\title{
Complete Revascularization of Stable STEMI Patients Offers a Significant Benefit if Done During the Index PCl, but Not if It's Done as a Staged Procedure
}

\section{Roberto C Cerrud- \\ Rodriguez $\mathbb{D}^{\prime}$ \\ Syed Muhammad Ibrahim \\ Rashid' \\ Karlo A Wiley' \\ Maday Gonzalez' \\ Valeriia A Kosmacheva' \\ Isabella Castillero-Norato ${ }^{2}$ \\ Cornelia Rivera' \\ Pedro Villablanca ${ }^{3}$ \\ Jose Wiley'}

'Montefiore-Einstein Center for Heart and Vascular Care, Montefiore Medical Center, Albert Einstein College of Medicine, Bronx, NY, USA; ${ }^{2}$ Facultad de Medicina, Universidad de Panamá, Campus Octavio Méndez Pereira, Panama City, Republic of Panama; ${ }^{3}$ Department of Medicine, Division of Cardiology, Henry Ford Hospital, Detroit, MI, USA
Correspondence: Roberto C CerrudRodriguez

Montefiore-Einstein Center for Heart and Vascular Care, Montefiore Medical Center, Albert Einstein College of Medicine, III East 210th Street, Bronx, NY, 10467, USA

Tel +l 718-920-7929

Email robertocerrud@gmail.com
Background: Complete revascularization (CR) of hemodynamically stable STEMI improves outcomes when compared to culprit-only PCI. However, the optimal timing for $\mathrm{CR}$ (CR during index PCI [iCR] versus staged PCI [sCR]) is unknown. sCR is defined as revascularization of non-culprit lesions not done during the index procedure (mean 31.5 \pm 24.6 days after STEMI). Our goal was to determine whether iCR was the superior strategy when compared to sCR.

Methods: A systematic review of Medline, Cochrane, and Embase was performed for RCTs reporting outcomes of stable STEMI patients who had undergone CR. Only RCTs with a clearly defined timing of CR, for the classification into $\mathrm{iCR}$ and $\mathrm{SCR}$, and a follow-up of at least 12 months were included. Seven RCTs comprising 6647 patients (mean age:62.9 \pm 1.4 years, male sex:79.4\%) met these criteria and were included.

Results: After a mean follow-up of $25.1 \pm 9.4$ months, iCR was associated with a significant reduction in cardiovascular mortality (risk ratio [RR] $0.48,95 \%$ confidence interval [CI] $0.26-0.90, \mathrm{p}=0.02$, relative risk reduction [RRR] 52\%) and non-fatal reinfarctions (RR 0.42, 95\% CI $0.25-0.70, \mathrm{p}=0.001$, RRR: $58 \%$ ). sCR showed a significant reduction in non-fatal reinfarctions only (RR $0.68,95 \%$ CI $0.54-0.85, p=0.0008, R R R: 32 \%$ ). There was no difference in the safety outcome of contrast-induced nephropathy between groups.

Conclusion: iCR of stable STEMI patients is associated with a significant reduction in cardiovascular death and a trend towards reduction in all-cause mortality. These benefits are not seen in SCR. Both strategies are associated with a reduction in non-fatal reinfarctions.

Keywords: ST-segment elevation myocardial infarction, STEMI, percutaneous coronary intervention, PCI, staged revascularization, complete revascularization.

\section{Introduction}

Percutaneous coronary intervention (PCI) is the treatment of choice for ST-elevation myocardial infarction (STEMI), receiving a class I, Level of Evidence (LOE): A, recommendation in the $2013 \mathrm{ACCF} / \mathrm{AHA}$ guidelines for the management of STelevation myocardial infarction. ${ }^{1}$ Around $50 \%$ of patients presenting with STEMI have multivessel coronary artery disease (MVCAD), defined as $\geq 50 \%$ stenosis in a non-culprit coronary artery during index angiography. ${ }^{2}$ MVCAD in patients with STEMI carries a higher MACE risk than single vessel disease and as a result, many RCT's have focused on whether treatment of non-culprit lesions is beneficial. 
The 2013 guidelines, which gave a class III LOE $\mathrm{B}$ indication (meaning harm) to primary PCI of non-culprit MVCAD in stable STEMI patients, were derived from limited, and often conflicting, data. ${ }^{1}$ The subsequent publication of several randomized control trials (RCT) showed that complete revascularization (CR) of MVCAD in stable STEMI patients, either during index PCI (iCR) or as a staged procedure (sCR), might improve outcomes. ${ }^{3-5}$ This prompted a reassessment of the 2013 guidelines.

This process culminated with the publication of the 2015 ACC/AHA/SCAI Focused Update on Primary Percutaneous Coronary Intervention for Patients With ST-Elevation Myocardial Infarction, where CR was changed from a class III recommendation (harm) to a class IIb LOE B-R (moderate quality evidence from 1 or more RCTs). ${ }^{6}$

However, there was still hesitancy in adopting these guidelines into clinical practice due to the relatively small number of patients enrolled in previous RCTs, the lack of single hard outcomes reaching statistical significance (dependence on composite outcomes), and the absence of a recommendation on the exact timing of CR (iCR vs sCR). A recently published, large multicenter RCT, the COMPLETE trial (Complete Revascularization with Multivessel PCI for Myocardial Infarction), ${ }^{7}$ enrolled 4041 patients with a median follow-up of 3 years. It showed benefit in two coprimary composite outcomes (first coprimary composite of cardiovascular (CV) death or myocardial infarction (MI) (hazard ratio [HR] 0.74, $95 \%$ confidence interval $[\mathrm{CI}] 0.60-0.91, \mathrm{p}=0.004)$, and second coprimary composite outcome of cardiovascular (CV) death, MI or ischemia-driven revascularization (HR $0.51,95 \%$ CI $0.43-0.61, \mathrm{P}<0.001$ ) when $\mathrm{CR}$ strategy was performed. However, this study also failed to show significant benefit for CR with regards to individual hard outcomes of $\mathrm{CV}$ death (HR $0.93,95 \%$ CI $0.65-1.32$ ) or all-cause mortality (HR $0.91,95 \%$ CI $0.69-1.20)^{7}$

A recent meta-analysis by Pavasini et al showed a significant benefit of CR vs culprit-lesion only strategy in $\mathrm{CV}$ death (HR $0.62,95 \% \mathrm{CI} 0.39-0.97, \mathrm{p}=0.04$ ) and repeat MI (HR $0.65,95 \%$ CI $0.53-0.80, \mathrm{p}<0.0001$ ) but failed to demonstrate a significant benefit in all-cause mortality (HR 0.81, 95\% CI $0.60-1.10, \mathrm{p}=0.18){ }^{8}$ A previous meta-analysis and metaregression by Pasceri et al ${ }^{9}$ showed a significant total mortality benefit in CR compared to culprit-lesion only revascularization (Relative Risk [RR] 0.62, 95\% CI 0.39-0.97). However, that analysis included two RCTs (Hamza et al and HELP AMI) ${ }^{10,11}$ that are not generalizable due to a strict inclusion criteria (Hamza et al with diabetic patients only), ${ }^{11}$ or outdated technology (HELP AMI with heparin-coated stents). ${ }^{10}$
Given the aforementioned lack of clarity regarding the optimal timing for $\mathrm{CR}$ and its true effects on clinically relevant outcomes, we decided to perform a systematic review and meta-analysis of all available RCTs that met our criteria to try to ascertain if the timing of $\mathrm{CR}$ (either iCR or sCR when compared with a culprit-lesion only strategy) had any impact on single, hard outcomes of allcause mortality, CV death or non-fatal reinfarction). A secondary goal of this meta-analysis was to determine if there were any differences in safety outcomes of contrast-induced nephropathy between iCR and sCR.

\section{Methods}

The present meta-analysis was performed according to Cochrane Collaboration and Preferred Reporting Items for Systematic Reviews and Meta-Analyses (PRISMA) statements. $^{12}$ This meta-analysis was registered in PROSPERO with registration number CRD42020155116.

\section{Search Strategy}

We searched PubMed, Embase, and Cochrane Central Register of Clinical Trials (Cochrane Library, Issue 02, 2017) databases from January 2008 through November 2020 to identify RCTs comparing a CR vs culprit-lesion only strategy in stable patients presenting with STEMI.

We used the following terms: ("complete revascularization") AND ("STEMI" OR "ST-elevation myocardial infarction"). Language was restricted to papers in English only. The reference lists of identified articles were also exhaustively reviewed for additional sources.

\section{Eligibility Criteria}

Studies with the following characteristics were considered eligible: (A) RCTs comparing CR vs culprit-lesion only in stable patients with STEMI; (B) clearly identified the timing of the CR strategy (either iCR or sCR); (C) compared the event rates of all-cause mortality, $\mathrm{CV}$ death and nonfatal reinfarction between the two groups; (D) compared the rates of strokes and CIN between groups; (F) had a follow-up period of at least 12 months.

Case reports, editorials, reviews, non-randomized studies and expert opinions were excluded from our analysis. Abstracts presented in major international conferences that have not been published as full papers were not considered in our analysis. 


\section{Primary Outcome and Composite Safety Outcome}

The primary outcomes of this study were (A) All-cause mortality (B) CV death, and (C) non-fatal reinfarction. The safety outcome was contrast-induced nephropathy (CIN).

$\mathrm{CV}$ death was defined as all deaths with a clear cardiovascular or unknown cause. Reinfarction was defined using the Fourth Universal Definition of Myocardial Infarction. ${ }^{13}$ $\mathrm{CIN}$ was defined as an elevation of serum creatinine of $\geq 25 \%$ or $\geq 0.5 \mathrm{mg} / \mathrm{dl}(44 \mu \mathrm{mol} / \mathrm{l})$ from baseline within $48 \mathrm{~h}^{14}$

\section{Data Extractions and Quality Appraisal}

Two investigators (R.C.C.R and S.M.I.R.) independently screened all titles, abstracts and manually searched the full text versions of all relevant studies that fulfilled the inclusion criteria. References of the retrieved articles were independently reviewed for further identification of potentially relevant studies. Disagreements were resolved by consensus after discussion (R.C.C.R and S.M.I.R.). We extracted characteristics of each study including methodology and baseline patient characteristics, CV deaths, non-fatal reinfarction, stroke rate, and CIN rate. If the abovementioned information was not readily available in the written article, the principal investigator of that particular study was contacted to supply pertinent information.

\section{Quality Assessment}

The quality and reporting of the included RCTs were assessed using the Cochrane Risk of Bias Tool. ${ }^{15}$ Six categories were included in the analysis [A] Selection bias: systematic differences between baseline characteristics of the groups that are compared; [B] Performance bias: systematic differences between groups in the care that is provided, or in exposure to factors other than the interventions of interest; [C] Detection bias: systematic differences between groups in how outcomes are determined. Blinding of outcome assessors may reduce the risk that knowledge of which intervention was received, rather than the intervention itself, affects outcome measurement; [D] Attrition bias: systematic differences between groups due to withdrawals from a study. Withdrawals from the study lead to incomplete outcome data; [E] Reporting bias: systematic differences between reported and unreported findings; $[\mathrm{F}]$ Other biases: other sources of bias that are relevant only in certain circumstances. Quality of the included RCTs was summarized visually.

\section{Statistical Analysis}

Descriptive statistics are presented as number of cases (n) for dichotomous and categorical variables. Statistical analysis was performed in line with recommendations from the Cochrane Collaboration and PRISMA guidelines, using Review Manager (RevMan version 5.4, the Cochrane Collaboration, 2020). ${ }^{15}$ Heterogeneity was assessed using the $\mathrm{I}^{2}$ statistics, which is the proportion of total variation observed among the studies attributable to differences between studies rather than sampling error (chance). Data were summarized across groups using the Mantel-Haenszel Risk Ratio (RR) Fixed-Effect model if $\mathrm{I}^{2}<25 .{ }^{16} \mathrm{We}$ considered $\mathrm{I}^{2}<25 \%$ as low and $\mathrm{I}^{2} \geq 75 \%$ as high. The Random-Effects Model was used if $\mathrm{I}^{2} \geq 25 \%$. Publication bias was estimated visually by funnel plots. ${ }^{15}$

\section{Results}

A total of 204 studies were identified using the specified search criteria (Figure 1). After evaluation of these studies based on titles and abstracts, $10 \mathrm{RCTs}$ were further analyzed in their full-text version, 3 of which were excluded to result in 7 RCTs that fulfilled all inclusion criteria. These 7 RCTs incorporated a total of 6647 participants (79.4\% male, average age $62.9 \pm 1.4$ years, mean follow-up period 25.1 \pm 9.4 months). Other RCTs were excluded due to a lack of information relevant to our study questions, narrow population (Hamza et al RCT of diabetic patients only ${ }^{11}$ limiting generalizability, outdated technology that is no longer routinely used (HELP-AMI) with its heparincoated stents), ${ }^{10}$ or because of insufficient follow-up (less than 12 months).

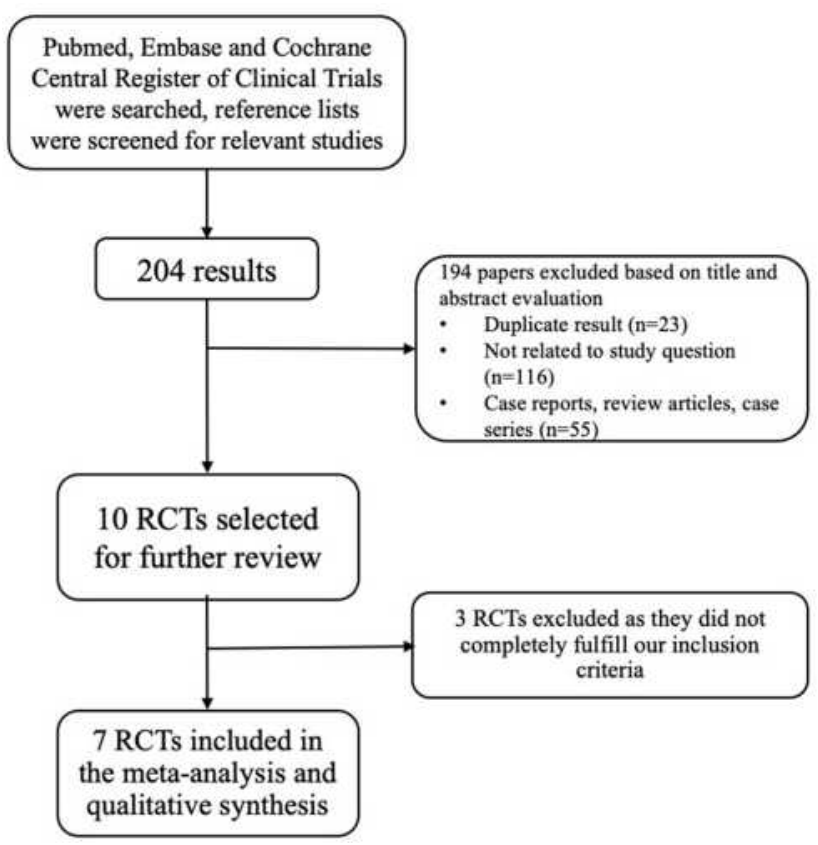

Figure I Study selection. Process of study selection. Abbreviation: RCT, randomized control trial. 


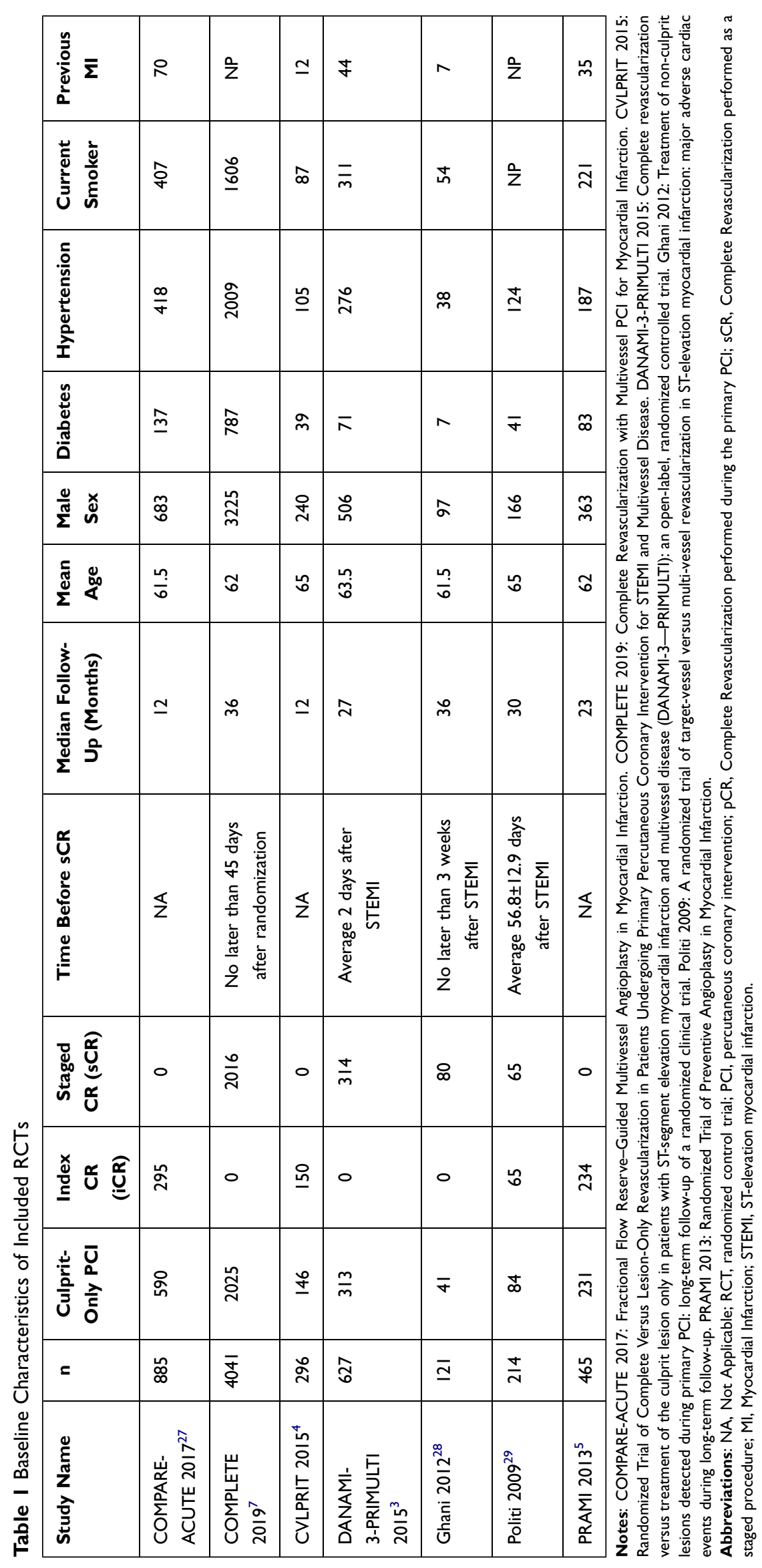




\section{Characteristics of Included Studies}

The baseline characteristics of the included trials are summarized in Table 1. Complete revascularization during index PCI was undertaken in 744 participants (11.2\%), whereas CR was done as a staged procedure in 2475 participants $(37.2 \%)$. Culprit-vessel only PCI was done in 3430 participants $(51.6 \%)$. The mean age was 62.9 \pm 1.4 years; $79.4 \%$ were males. The mean follow-up period was $25.1 \pm 9.4$ months.

\section{Quality Assessment and Publication Bias}

Funnel plots did not suggest publication bias for the selected outcomes (Figure 2). All the RCTs included in this meta-analysis had good methodological quality indicating "low risk of bias" (Figure 3).

\section{Impact of Complete Revascularization on All-Cause Mortality}

There was a non-significant, but remarkable trend towards an all-cause mortality benefit in the iCR group when compared with the culprit-only group: iCR (RR 0.63, 95\% CI $0.40-1.00, \mathrm{p}=0.05)$. No significant difference was seen in the $\mathrm{sCR}$ group when compared with the culprit-only group (RR 0.92, 95\% CI 0.57-1.49, p=0.75) (Figure 4).

\section{Impact of Complete Revascularization on Cardiovascular Mortality}

There was a statistically significant reduction in cardiovascular mortality in the iCR group, when compared to the culprit-lesion only group (RR 0.48 , 95\% CI $0.26-0.90$, $\mathrm{p}=0.02$ ), with a relative risk reduction (RRR) of $52 \%$ (Figure 5). There was no benefit seen in the sCR group (RR 0.73, 95\% CI 0.38-1.41, p=0.35).

\section{Impact of Complete Revascularization on Non-Fatal Reinfarction}

We found a statistically significant reduction in non-fatal reinfarctions in both complete revascularization groups, regardless of timing, when compared with the culpritonly strategy. The iCR group (RR 0.42, 95\% CI 0.25-0.70,
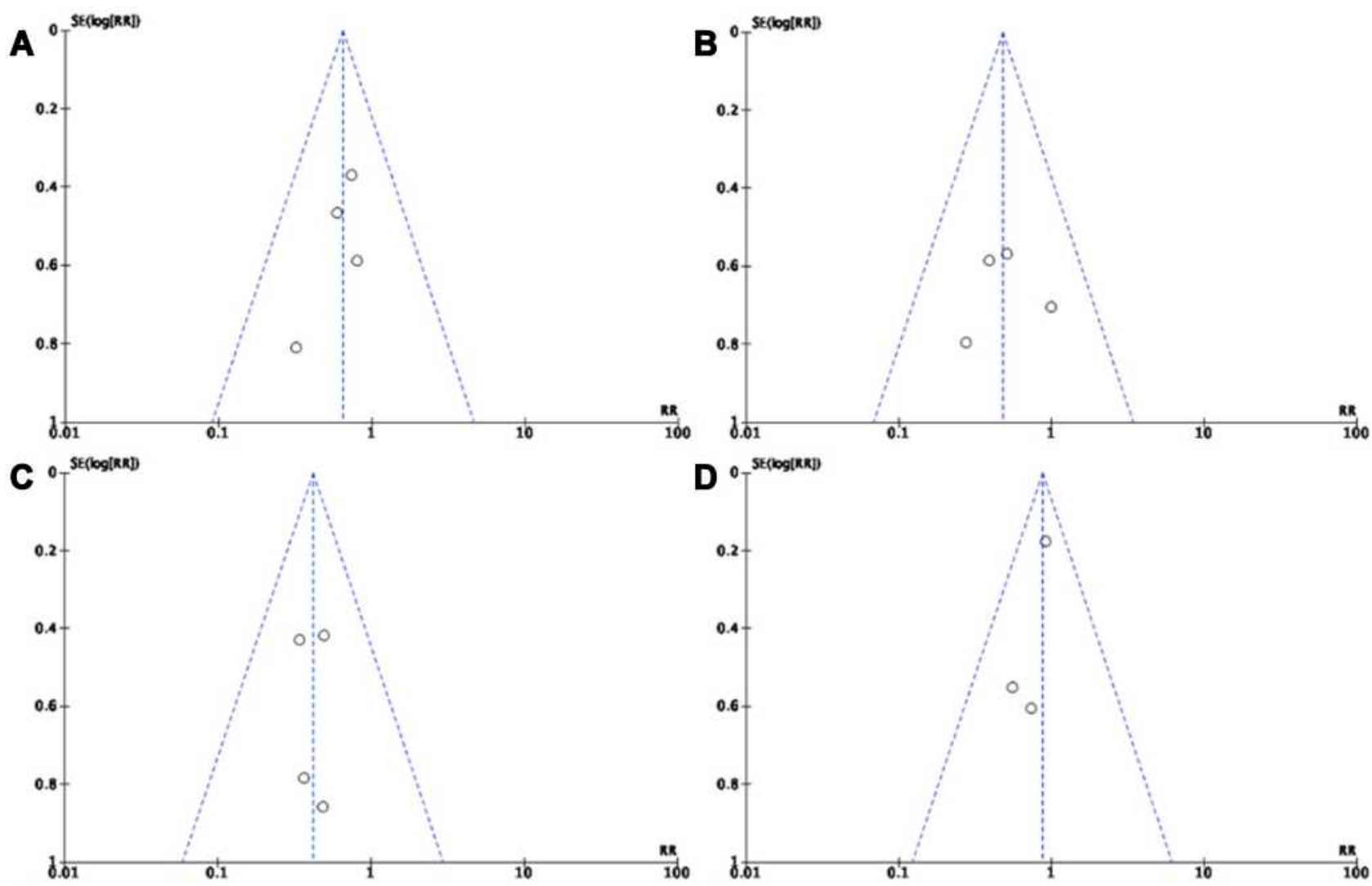

Figure 2 Funnel Plots - (A) all-cause mortality of complete revascularization during index $\mathrm{PCl}$ (B) cardiovascular mortality of staged complete revascularization (C) reinfarction events of complete revascularization during index $\mathrm{PCl}(\mathbf{D})$ reinfarction events of complete revascularization during staged complete revascularization. Primary complete revascularization: Revascularization done at the time of primary percutaneous catheter intervention (PCl). Staged complete revascularization: Revascularization done at a different time than the primary $\mathrm{PCl}$. 


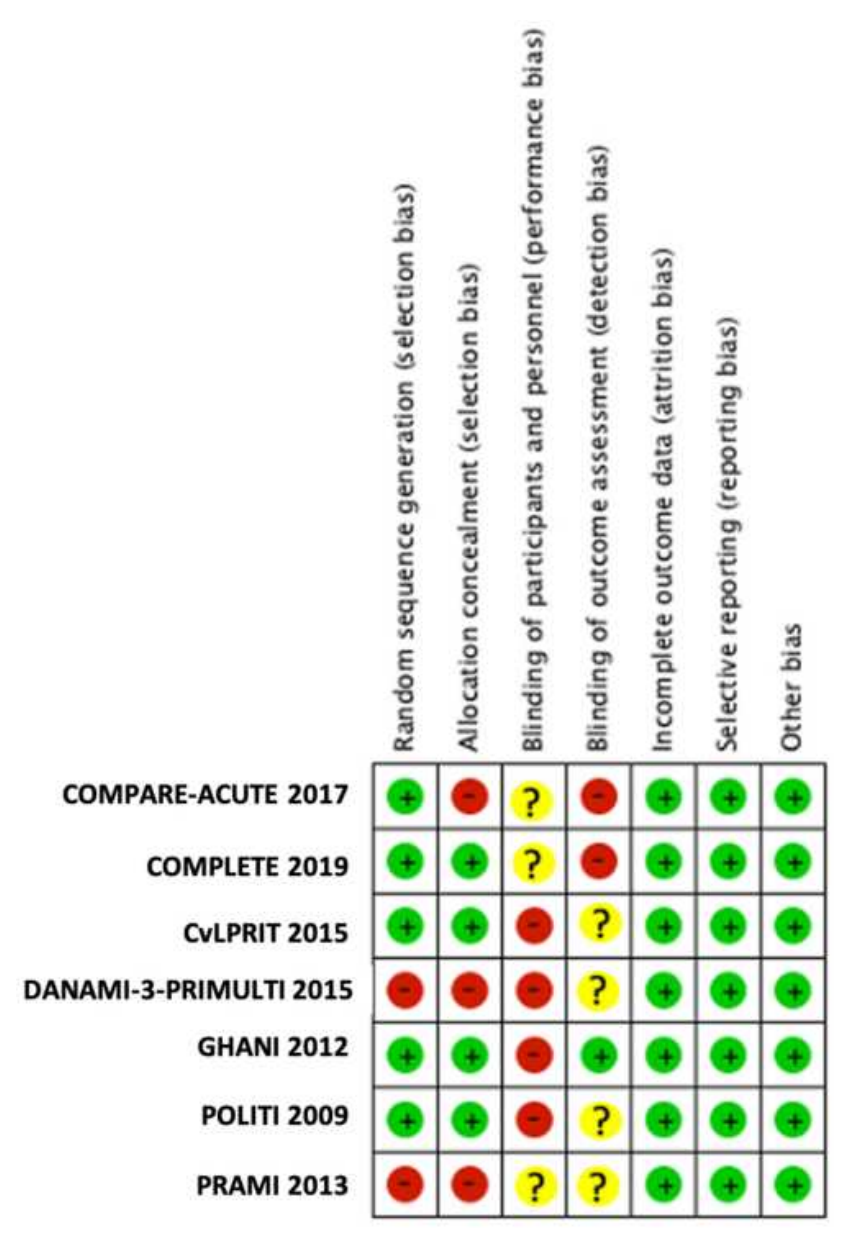

Figure 3 Risk of bias summary: review authors' judgements about each risk of bias item for each included study. COMPARE-ACUTE 2017: Fractional Flow ReserveGuided Multivessel Angioplasty in Myocardial Infarction. COMPLETE 2019: Complete Revascularization with Multivessel $\mathrm{PCl}$ for Myocardial Infarction. CVLPRIT 2015: Randomized Trial of Complete Versus Lesion-Only Revascularization in Patients Undergoing Primary Percutaneous Coronary Intervention for STEMI and Multivessel Disease. DANAMI-3-PRIMULTI 2015: Complete revascularization versus treatment of the culprit lesion only in patients with ST-segment elevation myocardial infarction and multivessel disease (DANAMI3-PRIMULTI): an open-label, randomized controlled trial. Ghani 2012: Treatment of non-culprit lesions detected during primary $\mathrm{PCl}$ : long-term follow-up of a randomized clinical trial. Politi 2009: A randomized trial of target-vessel versus multi-vessel revascularization in ST-elevation myocardial infarction: major adverse cardiac events during long-term follow-up. PRAMI 2013: Randomized Trial of Preventive Angioplasty in Myocardial Infarction.

$\mathrm{p}=0.001$ ), with a relative risk reduction (RRR) of $58 \%$ (Figure 6), whereas the sCR group (RR 0.68, 95\% CI $0.54-0.85, \mathrm{p}=0.0008$ ) with a RRR of $32 \%$. In the $\mathrm{sCR}$ cohort, the statistical weight of the COMPLETE trial is responsible for the significant decrease in reinfarctions.

\section{Safety Outcome of Contrast-Induced Nephropathy}

There was no significant difference in outcomes between the complete revascularization groups and the culprit-lesion only group: iCR (RR 0.67, 95\% CI $0.16-2.82, \mathrm{p}=0.58)$ and sCR (RR 0.83, 95\% CI 0.83-2.17, $\mathrm{p}=0.23$ ) (Figure 7).

\section{Discussion}

Complete revascularization of MVCAD in patients who present with a hemodynamically stable STEMI has been shown to be beneficial when compared with a culpritvessel only strategy in previously published metaanalyses. ${ }^{8,9}$ In a recently published meta-analysis by Atti et al, complete revascularization was associated with a significantly decreased reinfarction rate (RR $0.69,95 \%$ CI $0.50-0.95$ ) and repeat revascularization (RR $0.34,95 \%$ CI $0.25-0.44$ ) with no benefit in the other studied efficacy outcomes (all-cause mortality and cardiovascular mortality). ${ }^{17}$ However, the vast majority of the previous meta-analyses pooled together data from papers and populations that underwent complete revascularization during the index PCI as well as those who did so as a staged procedure after the culprit lesion was treated, without distinction regarding timing.

We performed this meta-analysis to determine whether the timing of CR (iCR vs SCR), has an impact on cardiovascular mortality, non-fatal reinfarction, and all-cause mortality. With our findings, we have demonstrated that iCR is superior to $\mathrm{SCR}$ because it lowers $\mathrm{CV}$ mortality and shows a beneficial, yet non-significant trend in all-cause mortality, when compared to culprit-only revascularization in stable patients presenting with STEMI. This is important and relevant for a number of reasons.

Firstly, it challenges current day practice in which sCR is more commonly performed than iCR. In a large cohort study by Secemsky et al using the National Cardiovascular Data Registry CathPCI Registry from the third quarter of 2009 to the first quarter of $2018,{ }^{18}$ multivessel PCI was performed in $n=138,380$ STEMI patients. Of these, $30.8 \%$ $(n=42,629)$ had multivessel PCI performed during the index procedure, $31.6 \%(\mathrm{n}=43,696)$ were done as staged procedures during the index admission and $37.6 \%$ $(\mathrm{n}=52,055)$ had multivessel PCI done within 45 days of discharge. ${ }^{18}$ The same fact can be observed in our metaanalysis, where $77 \%$ of the patients underwent a staged complete revascularization while only $23 \%$ underwent complete revascularization during the index PCI.

sCR is likely popular because high quality data regarding the optimal timing does not exist at this time, as even a recent review article by Bossard and Mehta puts in evidence - the interventional community agrees on the 


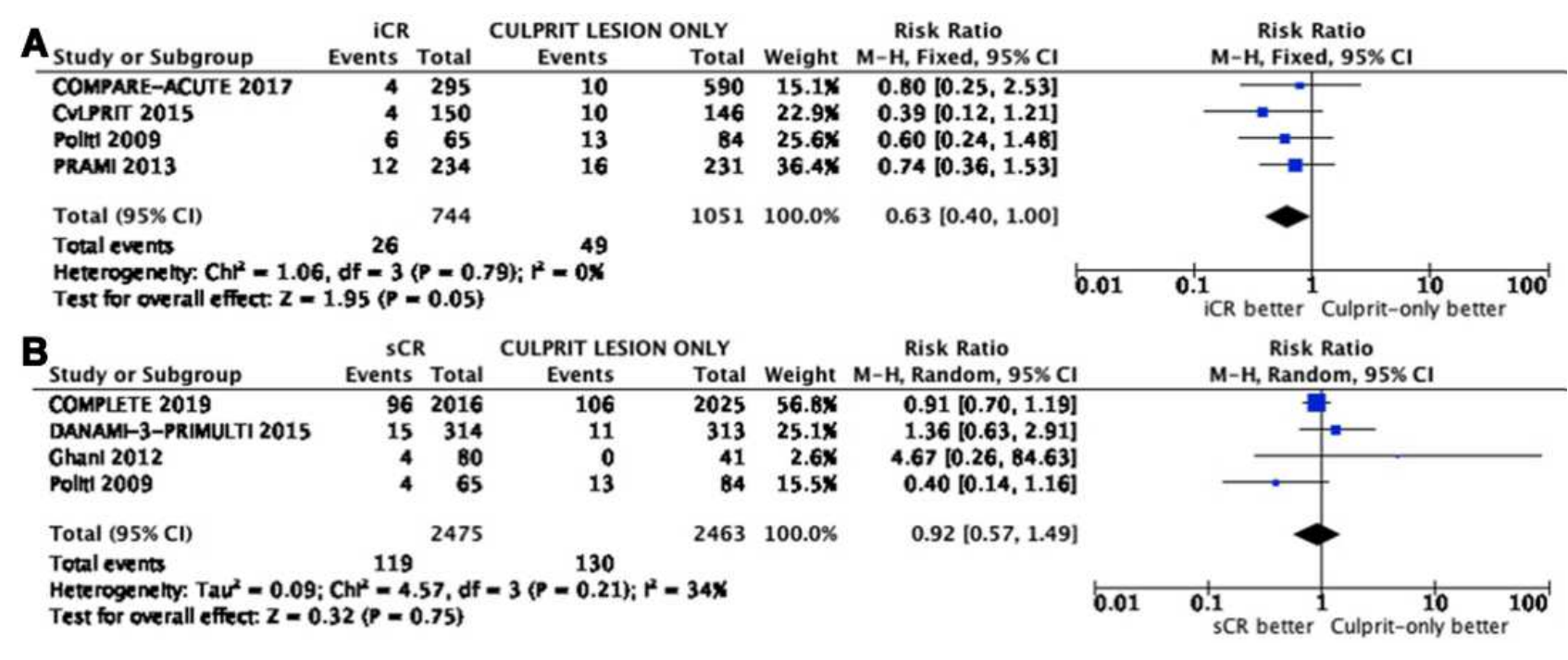

Figure 4 Forest Plot - all-cause mortality (A) complete revascularization during index PCl (B) staged complete revascularization. Diamond indicates overall summary estimate for the analysis (width of the diamond represents the $95 \% \mathrm{Cl}$ ); width of the shaded square, size of the population. Fixed-effect model was used for this outcome during index $\mathrm{PCl}$ as $\mathrm{I}^{2}<25$; random effects model was used for the staged complete revascularization outcome given $\mathrm{I}^{2} \geq 25$.

Abbreviations: $\mathrm{Cl}$, confidence interval; $\mathrm{MH}$, Mantel-Haenszel; Complete: complete revascularization strategy; Culprit-only, culprit-only revascularization strategy; PCI, percutaneous coronary intervention; iCR, complete revascularization during index $\mathrm{PCl}$; sCR, staged complete revascularization.

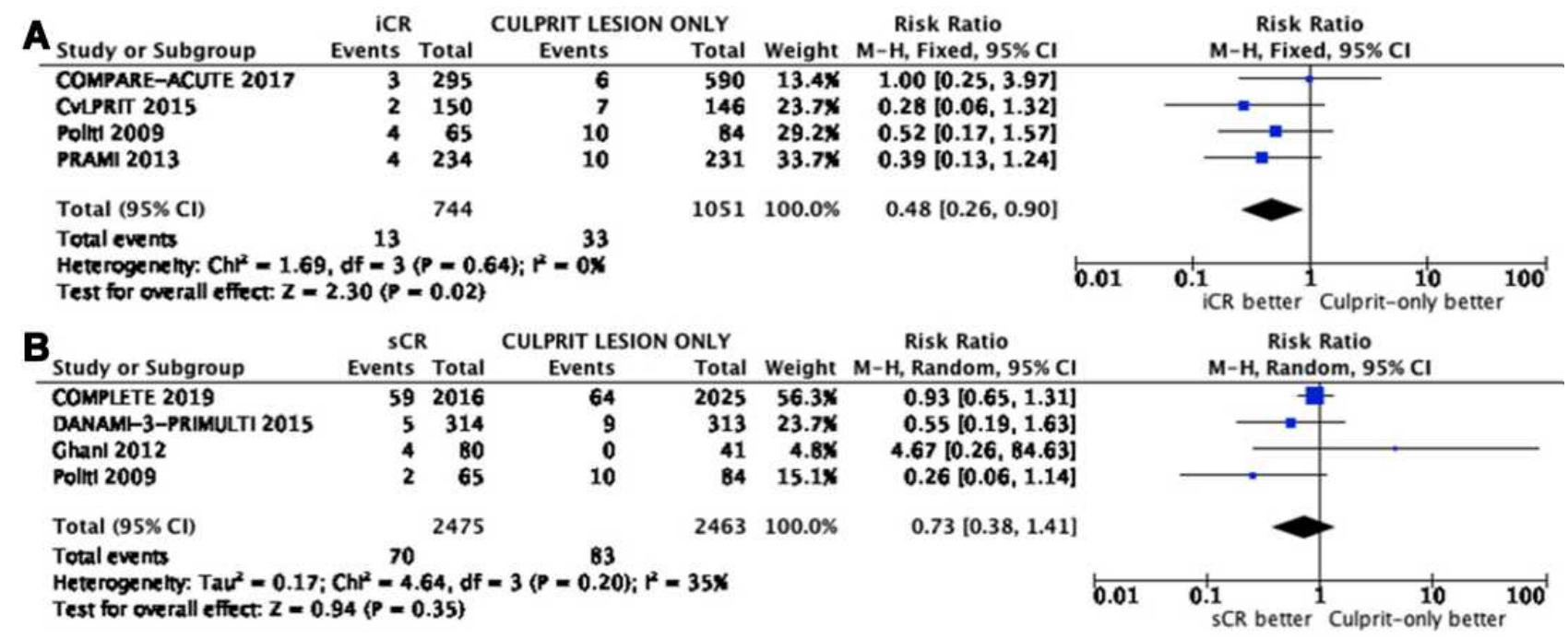

Figure 5 Forest Plot - cardiovascular mortality $(\mathbf{A})$ complete revascularization during index $\mathrm{PCl}(\mathbf{B})$ staged complete revascularization. Diamond indicates overall summary estimate for the analysis (width of the diamond represents the $95 \% \mathrm{Cl}$ ); width of the shaded square, size of the population. Fixed-effect model was used for this outcome during index $\mathrm{PCl}$ as $\mathrm{I}^{2}<25$; random effects model was used for the staged complete revascularization outcome given $\mathrm{I}^{2} \geq 25$.

Abbreviations: $\mathrm{Cl}$, confidence interval; $\mathrm{MH}$, Mantel-Haenszel; Complete, complete revascularization strategy; Culprit-only, culprit-only revascularization strategy; PCI, percutaneous coronary intervention; iCR, complete revascularization during index $\mathrm{PCl}$; sCR, staged complete revascularization.

benefits of performing a complete revascularization of this patient population, but there is a lack of clear guidance regarding the best timing. ${ }^{19}$ Also, performing a sCR vs $\mathrm{iCR}$ is believed in routine clinical practice to lower the risk of CIN, a belief that is refuted by the findings of our metanalysis, which showed that no significant difference exists in CIN between the two strategies.
Next, our analysis shows a trend towards benefit in allcause mortality when iCR is compared to culprit only (RR $0.63,95 \%$ CI $0.40-1.00, \mathrm{p}=0.05$ ) which is not seen when sCR was compared to culprit only (RR $0.92,95 \%$ CI 0.57-1.49, $\mathrm{p}=0.75$ ). To date, no RCT has ever shown an all-cause mortality benefit with complete revascularization, possibly because no trials have previously been 


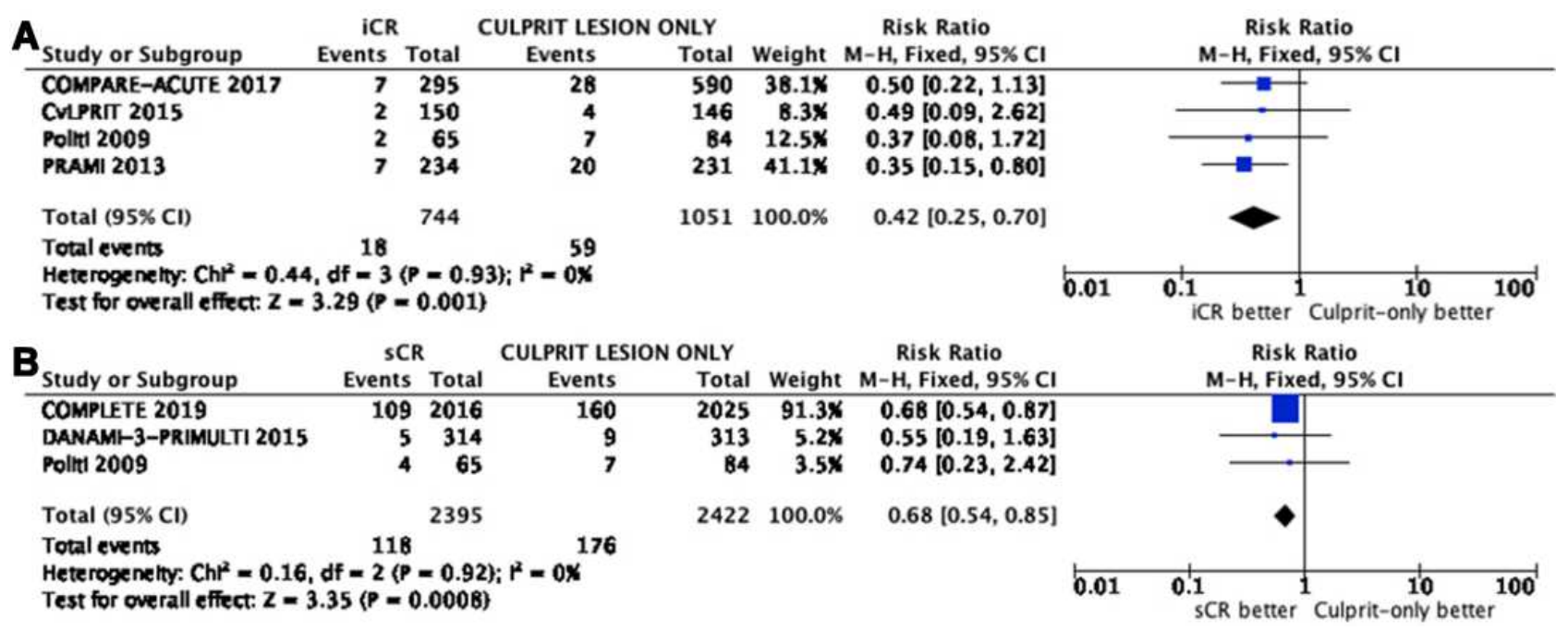

Figure 6 Forest Plot - non-fatal reinfarction (A) complete revascularization during index $\mathrm{PCl}(\mathbf{B})$ staged complete revascularization. Diamond indicates overall summary estimate for the analysis (width of the diamond represents the $95 \% \mathrm{Cl}$ ); width of the shaded square, size of the population. Fixed-effect model was used in both outcomes as $\mathrm{I}^{2}<25$

Abbreviations: $\mathrm{Cl}$, confidence interval; $\mathrm{MH}$, Mantel-Haenszel; Complete, complete revascularization strategy; Culprit-only, culprit-only revascularization strategy; PCl, percutaneous coronary intervention; iCR, complete revascularization during index $\mathrm{PCl}$; sCR, staged complete revascularization.

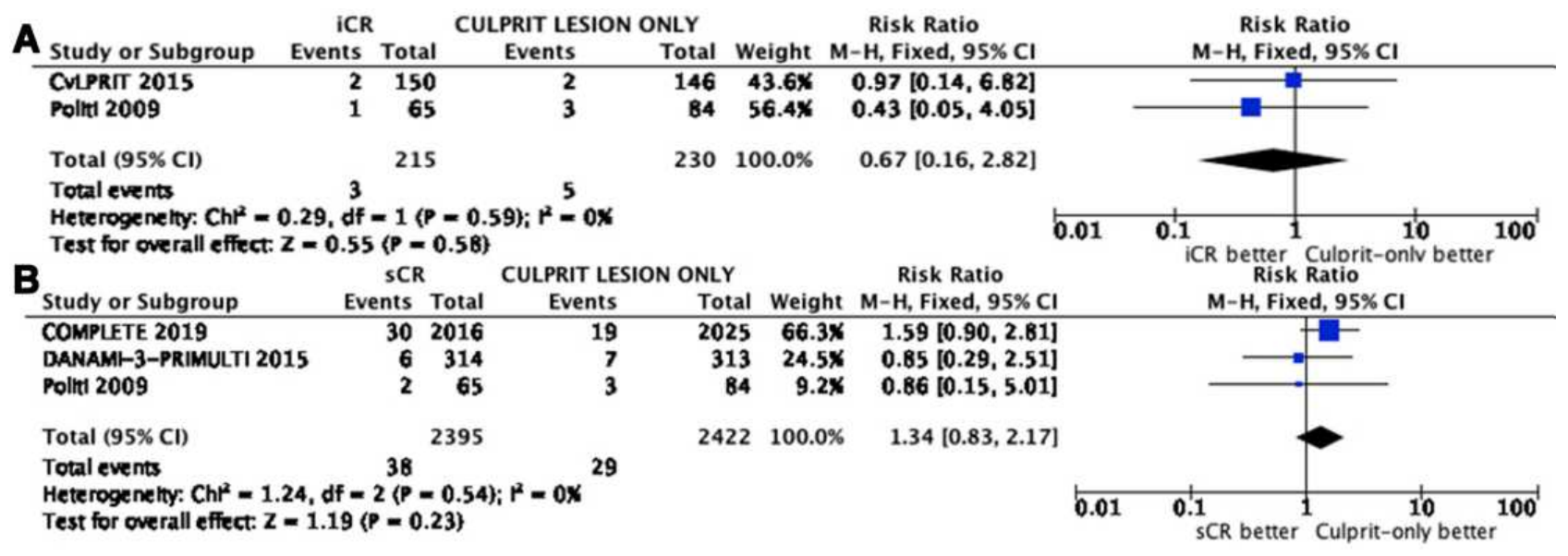

Figure 7 Forest Plot - safety outcome of contrast-induced nephropathy (A) complete revascularization during Index PCl (B) staged complete revascularization. Diamond indicates overall summary estimate for the analysis (width of the diamond represents the $95 \% \mathrm{Cl}$ ); width of the shaded square, size of the population. Fixed-effect model was used in both outcomes as $I^{2}<25$.

Abbreviations: $\mathrm{Cl}$, confidence interval; $\mathrm{MH}$, Mantel-Haenszel; Complete, complete revascularization strategy; Culprit-only, culprit-only revascularization strategy; PCl, percutaneous coronary intervention.

powered to detect this difference. The $\mathrm{CR}$ arm in the COMPLETE trial consisted only of SCR (acknowledged by the authors as a limitation of the trial) ${ }^{7}$ and its results were consistent with our meta-analysis as it failed to show a benefit in CV mortality or all-cause mortality. ${ }^{7}$

Lastly, complete revascularization during the index PCI has the potential to be more convenient for the patient, depending on their clinical condition, and might even potentially decrease healthcare costs. $\mathrm{SCR}$ is often done later during the index hospitalization, which increases the length of stay and potentially further increases healthcare costs. ${ }^{20}$ Alternatively, patients are discharged and electively readmitted for the $\mathrm{sCR}$, which can be inconvenient for the patient. Furthermore, this can be challenging to coordinate if patients have poor socioeconomic backgrounds or low health literacy. ${ }^{21}$

Our meta-analysis updates the findings presented by Pasceri et al, which tried to ascertain the best timing of complete revascularization to achieve optimal outcomes. ${ }^{9}$ They used a composite outcome of death and MI and determined that complete revascularization 
during index PCI was associated with a significant benefit in the primary composite outcome. ${ }^{9}$ A recent metaanalysis by Bainey et $\mathrm{al}^{22}$ compared the outcomes of complete revascularization of STEMI patients compared with a culprit-only strategy, showing significant benefit in cardiovascular death (Odds Ratio [OR], 0.69 [95\% CI, 0.48-0.99]; $\mathrm{P}=0.05$ ) and in the composite outcome of cardiovascular death and new MI (OR, 0.69 [95\% CI, 0.55-0.87]; $\mathrm{P}=0.001)$. However, this paper did not study the differences in outcomes between complete revascularization during primary PCI vs complete revascularization as a staged procedure. ${ }^{22}$

The difference in $\mathrm{CV}$ death and mortality might be due to the early occurrence of MACE events, many of which have been found to occur within the first two to three weeks after index revascularization. ${ }^{4}$ This period is often shorter than the average time to sCR after the index PCI seen in clinical practice. Another hypothesis is that the iCR can improve perfusion to hibernating myocardium and areas of watershed infarction sooner, leading to improved LV function and subsequently, improved clinical outcomes. ${ }^{23}$ Of note, a pilot study assessing the usefulness of the SYNTAX II (SII) score in patients presenting with a STEMI and cardiogenic shock showed that SII was superior to SYNTAX score by using a receiver-operator curve, with the 2 higher tertiles of SII having a worse in-hospital mortality that the lower tertile. ${ }^{24}$

Despite the findings above that suggest iCR is superior, we do identify one practical limitation in performing iCR: catheterization lab schedule. There might be scenarios where multiple emergent cases require the attention of the interventionalist and from a real-world, cath lab logistics perspective it is simply more feasible to perform $\mathrm{SCR}$.

\section{Limitations}

Our meta-analysis has several limitations: the included studies had different inclusion and exclusion criteria, the majority of the patients enrolled into the studies were male, which might cause them to not represent the true effect of either strategy on female sex patients, and race, a strong predictor of severity of heart disease, with black women faring particularly worse and suffering more severe CAD than other sex and ethnic groups. ${ }^{25}$

Lastly, this meta-analysis included RCTs with hemodynamically stable patients only and these results cannot be extrapolated to STEMI patients presenting with cardiogenic shock, who do not benefit from a iCR strategy. ${ }^{26}$

\section{Conclusion}

Complete revascularization during index PCI of stable STEMI patients is associated with a statistically significant reduction in cardiovascular death and a non-significant trend towards a reduction in all-cause mortality compared to culprit-lesion-only revascularization, at $25.1 \pm 9.4$ months follow-up. These benefits are not seen in staged complete revascularization. Both strategies are associated with a reduction in non-fatal reinfarctions and do not have a significant difference in CIN rates. A RCT comparing iCR to sCR with sufficient statistical power is needed to confirm our findings.

\section{Abbreviations}

$\mathrm{CI}$, confidence interval; RR, risk ratio; RRR, relative risk reduction; STEMI, ST-segment elevation myocardial infarction; PCI, percutaneous coronary intervention; CR, complete revascularization; CIN; contrast-induced nephropathy; MVCAD, multivessel coronary artery disease; CAD, coronary artery disease; RCT, randomized control trial.

\section{Funding}

This research did not receive any specific grant from funding agencies in the public, commercial, or not-forprofit sectors.

\section{Disclosure}

All authors report no conflicts of interest.

\section{References}

1. O'Gara PT, Kushner FG, Ascheim DD, et al. 2013 ACCF/AHA guideline for the management of ST-elevation myocardial infarction: executive summary: a report of the American College of Cardiology Foundation/American Heart Association Task Force on Practice Guidelines. J Am Coll Cardiol. 2013;61(4):485-510.

2. Park DW, Clare RM, Schulte PJ, et al. Extent, location, and clinical significance of non-infarct-related coronary artery disease among patients with ST-elevation myocardial infarction. JAMA. 2014;312 (19):2019-2027. doi:10.1001/jama.2014.15095

3. Engstrom T, Kelbaek H, Helqvist S, et al. Complete revascularisation versus treatment of the culprit lesion only in patients with ST-segment elevation myocardial infarction and multivessel disease (DANAMI-3-PRIMULTI): an open-label, randomised controlled trial. Lancet. 2015;386(9994):665-671. doi:10.1016/S0140-6736(15)60648-1

4. Gershlick AH, Khan JN, Kelly DJ, et al. Randomized trial of complete versus lesion-only revascularization in patients undergoing primary percutaneous coronary intervention for STEMI and multivessel disease: the CvLPRIT trial. J Am Coll Cardiol. 2015;65(10):963-972. doi:10.1016/j.jacc.2014.12.038

5. Wald DS, Morris JK, Wald NJ, et al. Randomized trial of preventive angioplasty in myocardial infarction. $N$ Engl $J$ Med. 2013;369 (12):1115-1123. doi:10.1056/NEJMoa1305520 
6. Levine GN, Bates ER, Blankenship JC, et al. 2015 ACC/AHA/SCAI Focused Update on Primary Percutaneous Coronary Intervention for Patients With ST-Elevation Myocardial Infarction: an Update of the 2011 ACCF/AHA/SCAI Guideline for Percutaneous Coronary Intervention and the 2013 ACCF/AHA Guideline for the Management of ST-Elevation Myocardial Infarction. $J$ Am Coll Cardiol. 2016;67(10):1235-1250. doi:10.1016/j.jacc.2015.10.005

7. Mehta SR, Wood DA, Storey RF, et al. Complete revascularization with multivessel PCI for myocardial infarction. $N$ Engl J Med. 2019;381(15):1411-1421. doi:10.1056/NEJMoa1907775

8. Pavasini R, Biscaglia S, Barbato E, et al. Complete revascularization reduces cardiovascular death in patients with ST-segment elevation myocardial infarction and multivessel disease: systematic review and meta-analysis of randomized clinical trials. Eur Heart J. 2019. doi:10.1093/eurheartj/ehy504

9. Pasceri V, Patti G, Pelliccia F, et al. Complete revascularization during primary percutaneous coronary intervention reduces death and myocardial infarction in patients with multivessel disease: Meta-Analysis and Meta-Regression of Randomized Trials. JACC Cardiovasc Interv. 2018;11(9):833-843. doi:10.1016/j.jcin.2018.02.028

10. Di Mario C, Mara S, Flavio A, et al. Single vs multivessel treatment during primary angioplasty: results of the multicentre randomised HEpacoat for cuLPrit or multivessel stenting for Acute Myocardial Infarction (HELP AMI) Study. Int J Cardiovasc Intervent. 2004;6 (3-4):128-133. doi:10.1080/14628840310030441

11. Hamza M, Mahmoud N, Elgendy IY. A randomized trial of complete versus culprit-only revascularization during primary percutaneous coronary intervention in diabetic patients with acute ST elevation myocardial infarction and multi vessel disease. $J$ Interv Cardiol. 2016;29(3):241-247. doi:10.1111/joic.12293

12. Moher D, Liberati A, Tetzlaff J, Altman DG. Preferred reporting items for systematic reviews and meta-analyses: the PRISMA statement. PLoS Med. 2009;6(7):e1000097.

13. Thygesen K, Alpert JS, Jaffe AS, et al. Fourth Universal Definition of Myocardial Infarction (2018). J Am Coll Cardiol. 2018;72 (18):2231-2264. doi:10.1016/j.jacc.2018.08.1038.

14. Mohammed NMA, Mahfouz A, Achkar K, Rafie IM, Hajar R. Contrast-induced nephropathy. Heart Views. 2013;14(3):106-116. doi:10.4103/1995-705X.125926

15. Page MH, Julian; Sterne, Jonathan. Assessing risk of bias due to missing results in a synthesis. In: Cochrane, editor. Cochrane Handbook for Systematic Reviews of Interventions version 61 (updated September 2020): Cochrane;2020.

16. DerSimonian R, Laird N. Meta-analysis in clinical trials. Controlled clinical trials. 1986;7:177-88.

17. Atti V, Gwon Y, Narayanan MA, et al. Multivessel versus culpritonly revascularization in STEMI and multivessel coronary artery disease: meta-Analysis of Randomized Trials. JACC Cardiovasc Interv. 2020;13(13):1571-1582. doi:10.1016/j.jcin.2020.04.055

18. Secemsky EA, Butala N, Raja A, et al. Temporal changes and institutional variation in use of percutaneous coronary intervention for ST-elevation myocardial infarction with multivessel coronary artery disease in the United States: an NCDR Research to Practice Project. JAMA Cardiol. 2020. doi:10.1016/S0735-1097(20)31755-1.
19. Bossard M, Mehta SR. Complete or incomplete revascularization for ST-segment elevation myocardial infarction: the PRAMI Trial to COMPLETE. Interv Cardiol Clin. 2020;9(4):433-440. doi:10.1016/ j.iccl.2020.06.001

20. Virani SS, Alonso A, Benjamin EJ, et al. Heart disease and stroke statistics - 2020 update: a report from the American Heart Association. Circulation. 2020;141(9):e139-e596.

21. Magnani JW, Mujahid MS, Aronow HD, et al. Health literacy and cardiovascular disease: fundamental relevance to primary and secondary prevention: a scientific statement from the American Heart Association. Circulation. 2018;138(2):e48-e74. doi:10.1161/ CIR.0000000000000579

22. Bainey KR, Engstrøm T, Smits PC, et al. Complete vs Culprit-Lesion-only revascularization for ST-segment elevation myocardial infarction: a systematic review and meta-analysis. JAMA Cardiol. 2020;5(8):1-9. doi:10.1001/jamacardio.2020.1251

23. Allman KC, Shaw LJ, Hachamovitch R, Udelson JE. Myocardial viability testing and impact of revascularization on prognosis in patients with coronary artery disease and left ventricular dysfunction: a meta-analysis. $J$ Am Coll Cardiol. 2002;39(7):1151-1158. doi:10.1016/S0735-1097(02)01726-6

24. Hayıroğlu M, Keskin M, Uzun AO, et al. Predictive value of SYNTAX score II for clinical outcomes in cardiogenic shock underwent primary percutaneous coronary intervention; a pilot study. Int $J$ Cardiovasc Imaging. 2018;34(3):329-336. doi:10.1007/ s10554-017-1241-9

25. Shaw LJ, Shaw RE, Merz CN, et al. Impact of ethnicity and gender differences on angiographic coronary artery disease prevalence and in-hospital mortality in the American College of Cardiology-National Cardiovascular Data Registry. Circulation. 2008;117(14):1787-1801. doi:10.1161/CIRCULATIONAHA.107.726562

26. Tuttle MK, Pinto DS. Culprit-only or complete revascularization for ST-elevation myocardial infarction in patients with and without shock. Interv Cardiol Clin. 2019;8(2):225-234. doi:10.1016/j. iccl.2019.01.001

27. Smits PC, Abdel-Wahab M, Neumann FJ, et al. Fractional flow reserve-guided multivessel angioplasty in myocardial infarction. $N$ Engl $J$ Med. 2017;376(13):1234-1244. doi:10.1056/ NEJMoa1701067

28. Ghani A, Dambrink JH, van ' $T$ Hof AW, Ottervanger JP, Gosselink AT, Hoorntje JC. Treatment of non-culprit lesions detected during primary PCI: long-term follow-up of a randomised clinical trial. Neth Heart J. 2012;20(9):347-353. doi:10.1007/s12471-0120281-y

29. Politi L, Sgura F, Rossi R, et al. A randomised trial of target-vessel versus multi-vessel revascularisation in ST-elevation myocardial infarction: major adverse cardiac events during long-term follow-up. Heart (British Cardiac Society). 2010;96(9):662-667. doi:10.1136/hrt.2009.177162
International Journal of General Medicine

\section{Publish your work in this journal}

The International Journal of General Medicine is an international, peer-reviewed open-access journal that focuses on general and internal medicine, pathogenesis, epidemiology, diagnosis, monitoring and treatment protocols. The journal is characterized by the rapid reporting of reviews, original research and clinical studies across all disease areas. The manuscript management system is completely online and includes a very quick and fair peer-review system, which is all easy to use. Visit http://www.dovepress.com/ testimonials.php to read real quotes from published authors. 